\title{
Communicating dosage instructions across cultural and linguistic barriers: Pharmacist-patient interactions in a South African antiretroviral clinic
}

Jennifer Watermeyer and Claire Penn

School of Human and Community Development, Department of Speech Pathology, University of the Witwatersrand, Private Bag 3, WITS, 2050, South Africa

E-mail: jennifer.watermeyer@wits.ac.za; claire.penn@wits.ac.za

\section{Introduction}

Over the past decade, HIV/AIDS has spread with astounding rapidity. The global introduction of antiretroviral drugs (ARVs) has decreased mortality rates and brought hope to many. However, the nature of ARV treatment is such that the patient must remain on it for the duration of life and research has shown that adherence levels of $80-95 \%$ are required to ensure treatment success (Gross et al. 2001; Paterson et al. 2000).

The advent of the HIV/AIDS epidemic has greatly increased the pharmacist's workload. Aside from dispensing drugs and monitoring for drug interactions and toxicities, pharmacists are also expected to provide counselling and advice that is tailored towards each patient's needs, as well as to ensure patient adherence to ARVs (Hardy 2005; Kansanaho 2006). However, pharmacists are rarely trained in communication skills (Salter et al. 2007).

Communication has been identified as a huge potential barrier in health settings and there is evidence of interference from psychosocial and HIV/Aids-related factors, as well as cultural and linguistic barriers. The manner in which a message is communicated by a health professional and understood by a patient is vital, because poor communication or misunderstanding of instructions may lead to non-adherence or difficulties in taking medicines (Sleath et al. 1999). In an age of globalisation, patient caseloads are increasingly multilingual and multicultural and the pharmacist must play a specific role in ensuring that communication with patients is successful despite cultural and linguistic barriers.

A growing body of research in South Africa has shown the ineffectiveness of communication across cultural and linguistic barriers, the many facets to cross-linguistic and cross-cultural communication, the pitfalls involved in using interpreters, and the need for further research in this area (Penn 2007). While there is a relatively large body of quantitative research investigating general communication skills required by pharmacists (De Young 1996), very little has been published that specifically examines the nature of interactions between patients and pharmacists, especially in a multicultural setting (Shah, King and Patel 2004). No 
literature exists which addresses communicative processes in an HIV or South African pharmacy context.

Pilnick's $(1998,1999,2001,2003)$ pioneering study conducted in the UK focused on the interactive features of pharmacist-caregiver interactions in a paediatric oncology clinic. Her analyses provide detailed insights into the nature of pharmacist-patient communication, counselling and advice giving. The interactional organisation of encounters between patients and pharmacists was found to be unique and differed from traditional templates for institutional talk between patients and other health professionals (Pilnick 2001). Pharmacists attempted to dominate interactions and they were often reluctant to question patients' knowledge or competence.

Dyck, Deschamps and Taylor (2005) examined Canadian pharmacy sessions and found that most information presented by pharmacists was given in a matter-of-fact, direct manner. Verification of patients' understanding of information was rare. Pharmacists tended to have their own agendas for consultations and expected specific responses rather than listening to patients' contributions. This so-called asymmetry was also noted by Skoglund, Isacson and Kjellgren (2003), who examined communication practices in Swedish pharmacy interactions. A pattern of pharmacist dominance and patient passivity was found to be present. What is noticeable in all of these studies is a lack of patient participation or collaboration and minimal acknowledgement of instructions provided by pharmacists, as well as minimal verification of patients' understanding of instructions.

In response to the identified lack of research in this field, as well as the urgent need to provide pharmacists with practical ways to improve communication with patients across cultural and linguistic barriers and thereby encourage patient adherence to ARVs, the authors conducted a sociolinguistic study of pharmacy interactions in a multicultural context in a South African HIV/Aids clinic pharmacy (Watermeyer 2008). The aim of this paper is to provide an overview of some of the findings of this study, including a description of various verbal and non-verbal strategies used by the pharmacists to present information and check understanding, as well as the joint construction of collaboration in interactions.

\section{Methods}

Data collection took place at a public hospital in North West Province, South Africa. The hospital's Wellness Clinic provides medical, pharmaceutical, nursing and counselling services to outpatients living with HIV/Aids. The pharmacy is situated in this Clinic and it comprises a separate consulting room with a waiting area outside. Two pharmacists, both first language Afrikaans speakers, work in the pharmacy. In between assisting patients, much of the pharmacists' time is spent on administrative and managerial tasks.

At the time of the study, 3,700 patients were enrolled in the ARV programme at the Clinic. Typically, patients are prescribed a first-line combination therapy ARV regimen, including two tablets to be taken twice daily and one tablet to be taken once daily, at a precise time. Antibiotics and vitamins are also routinely prescribed. Patients see the pharmacist for first and subsequent monthly visits during initiation of ARV therapy. During each visit, the pharmacist 
dispenses ARVs and other HIV-related medications, reviews the patient's health status, monitors adherence behaviours and presents or repeats dosage instructions.

Because this project involved research in a relatively unexplored field, the researchers employed broad selection criteria in order to gather data from a typical sample of patients seen by the pharmacists. A random sample of participants was obtained over a period of a week. Most patients were first language Setswana speakers who reported that they could speak and understand English and/or Afrikaans, although some were not proficient in either language. The sample included a range of education levels, language proficiencies, ages and visits to the pharmacy, as depicted in Table 1.

Table 1. Patient demographics

\begin{tabular}{|l|l|l|}
\hline \multicolumn{1}{|c|}{$\begin{array}{c}\text { Patient } \\
\text { demographic }\end{array}$} & \multicolumn{1}{|c|}{$\begin{array}{c}\text { Number of } \\
\text { patients }\end{array}$} \\
\hline Gender & Males & 9 \\
& Females & 17 \\
\hline Age & $20-30$ yrs & 9 \\
& $30-40$ yrs & 6 \\
& $40-50$ yrs & 7 \\
& $50-60$ yrs & 4 \\
\hline Education level & Tertiary (1-2 yrs) & 5 \\
& Finished high school & 4 \\
& Range from Gr 4 - Gr 11 & 16 \\
& Illiterate & 1 \\
\hline Language & Reportedly understand and speak & 16 \\
& English / Afrikaans & 10 \\
\hline Visits to pharmacy & Understand and speak Setswana only & 7 \\
& First visit & 4 \\
& Second visit & 6 \\
& Third visit & 9 \\
\hline
\end{tabular}

Most interactions were conducted in English, the second language of all participants. An interpreter is not available in the pharmacy, although on occasion caregivers or clinic staff are asked to interpret for patients.

After consent was obtained from participants, data collection comprised video recording of 26 pharmacist-patient interactions, post-interaction semi-structured interviews with participants and ethnographic observations in the pharmacy.

A descriptive, qualitative paradigm was employed for this study. A hybrid analytical approach was used, incorporating aspects of conversation analysis (CA) and discourse analysis (DA). Through detailed turn-by-turn analysis, CA seeks evidence of communication successes or failures from the context of the conversation and the responses of each conversation partner (ten Have 2000). DA allows for a focus on broader environmental influences such as HIV/Aids, the socio-political and economic context, culture and traditional beliefs, and how these impact on interactive processes (Wooffitt 2005). 
Salient verbal and non-verbal content in the interactions were transcribed using standard CA transcription conventions (Jefferson 2004) and Heath's (1986) system to transcribe non-verbal elements such as gaze, movements and gesture. Those parts of the interactions which were conducted in Setswana or Afrikaans were translated into English. After transcription, the interactional data were analysed using CA constructs such as turn taking, sequential organisation and repair sequences (Heritage 1993; Sacks 1984). CA revealed "micro" themes in each interaction and allowed the researcher to identify phases, overall outcomes and characteristics of the interaction. DA allowed the researchers to explore how several "macro" themes (social, linguistic, cultural, contextual and disease-related issues) in the data corpus impacted directly on the micro interactional features of the interactions. Interviews and ethnographic observations were analysed using techniques of Thematic Content Analysis (Aronson 1994). Emerging themes from these data were triangulated with results from analysis of the interactions to allow for comparison across the data (Patton 1990).

\section{Results}

Detailed analysis and comparisons across the data corpus revealed a precise structure in each interaction and a variety of mechanisms and communication processes which contributed towards collaboration in the interactions and understanding of information by patients across cultural and linguistic barriers. Various strategies and processes which pharmacists used to provide dosage instructions and information about ARVs to patients are discussed below with reference to relevant extracts from the data corpus.

\subsection{The structure of the interaction}

Distinct phases existed across most of the interactions, both in terms of content and organisational structure of the pharmacist-patient interaction. The structure of the interactions differed somewhat depending on whether it was a first or subsequent visit to the pharmacy, but all visits included elements such as provision of information about the drugs and dosage instructions, as well as strategies to check whether patients understood dosage instructions.

Pilnick's (2001) template for monolingual pharmacy interactions informed the examination of the structure and organisation of pharmacist-patient interactions in this study. The specific organisational structure of these multicultural interactions is described elsewhere (Watermeyer and Penn 2009c). After an initial opening and greeting, the interactions tended to move through cycles of approach or agenda-setting statements, delivery of information or instruction (often several different explanations of instructions), and patient responses (see Figure 1). The interactions also included regular response solicitations or tag questions (e.g. $n e ?^{1}$ and $o k$ ?) and checking of patients' understanding (e.g. do you understand? and tell me how you take the drugs), until the pharmacist was satisfied that the patient had understood the instructions. 


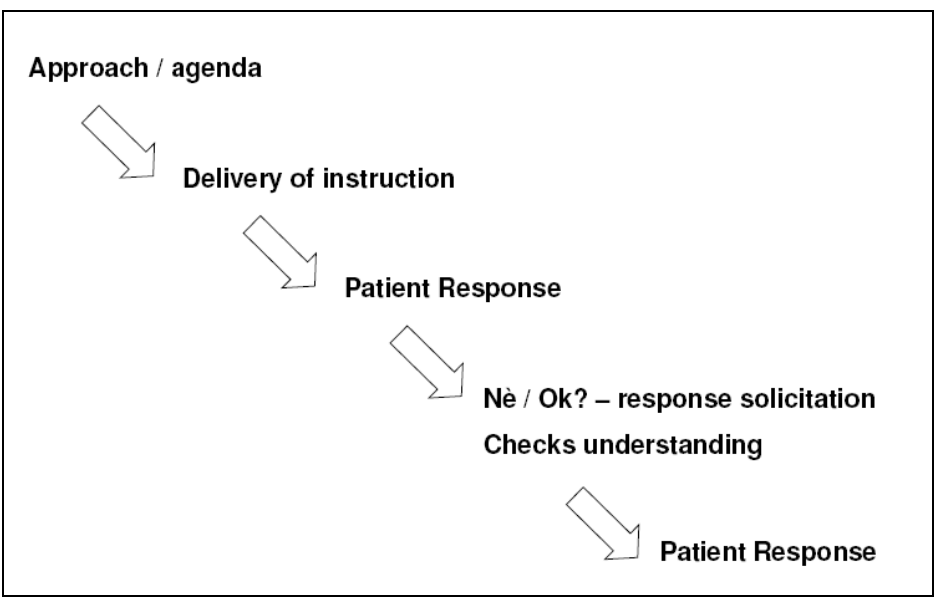

Fig. 1. Distinct cycles in the pharmacist-patient interaction

\subsection{Strategies for explanation of instructions}

The pharmacists employed a number of communication strategies which appeared to promote understanding of information and instructions by the patients and empowerment through gaining knowledge about ARV treatment. Some of these tools or strategies were used across the data corpus with most patients, while others were used with a few patients only. The selection of these strategies appeared to be based on a combination of the pharmacist's experience, intuition, skill and knowledge and/or assessment of the patient's needs. Patients responded to the use of these strategies by participating actively in interactions, collaborating with pharmacists and using some of the pharmacists' and their own communication strategies.

Strategies utilised by pharmacists included the following:

- Stipulation of the agenda for the session and provision of a running commentary during the interactions. An agenda-setting statement usually took the form of I'm going to explain to you now or We're going to start with the easy ones first. This served to focus patients' attention on the explanation, especially with patients who had poor concentration abilities.

- Verbal and non-verbal demarcation of ARVs from non-ARV medications. This strategy provided a clear link between the drugs and the corresponding explanations.

- Deliberate explanations of dosage instructions and provision of additional information about medications. Instructions were regularly related to patients' knowledge or stated concerns. Explanations were accompanied with non-verbal reinforcement using props (e.g. pill containers, boxes). Explanation of dosage instructions followed a specific format: the drug name was given, followed by an explanation of its purpose and dosage instructions.

- Repetition, reinforcement and summation of information and instructions. Pharmacists varied their explanations or initiated a more detailed demonstration of dosage instructions, depending on the demonstrated level of understanding by patients. For instance, if a patient did not appear to understand the instructions easily, pharmacists sometimes summarised what they had said in a slightly different manner, in order to provide varying explanations for the patient.

- Code switching (use of Setswana words and phrases), especially when giving complex instructions. 
- Repair strategies: evidence suggested sensitivity on the part of pharmacists to subtle misunderstandings, based on careful scrutiny and awareness of patients' verbal and nonverbal responses. Patients were sometimes able to identify when they did not understand and appeared comfortable to request clarification from pharmacists and initiate repair sequences. Some patients appeared to be more assertive than others in this regard.

In Extract 1, the pharmacist begins her explanation of the drugs with an agenda-setting statement (line 83): she will explain the drugs to the patient and start with the non-ARV drugs. She places a pile of pill packets (the non-ARV drugs) in front of the patient (line 84) and keeps the ARV drugs at the side of the table. By doing this, she not only sets the agenda for the following task, but also prepares the patient for the information which will follow and visually indicates which drug she will be talking about. She also clearly separates the ARV drugs from the non-ARV drugs and shows the patient each packet as she talks about the drug. The pharmacist's explanation of the drugs follows a specific format: first, she states the name of each drug, followed by an explanation of the purpose of the drug (line 85). Then she provides the dosage instruction for the drug (line 87). This methodical introduction is repeated across the data corpus with all drugs. The pharmacist waits for a response or acknowledgement from the patient after providing an instruction (line 87), but receives no reply (line 88). She therefore repeats the instruction and attaches a response solicitation, nè, to prompt a response from the patient (line 89). In line 90, the patient provides the response and the pharmacist continues with her explanation.

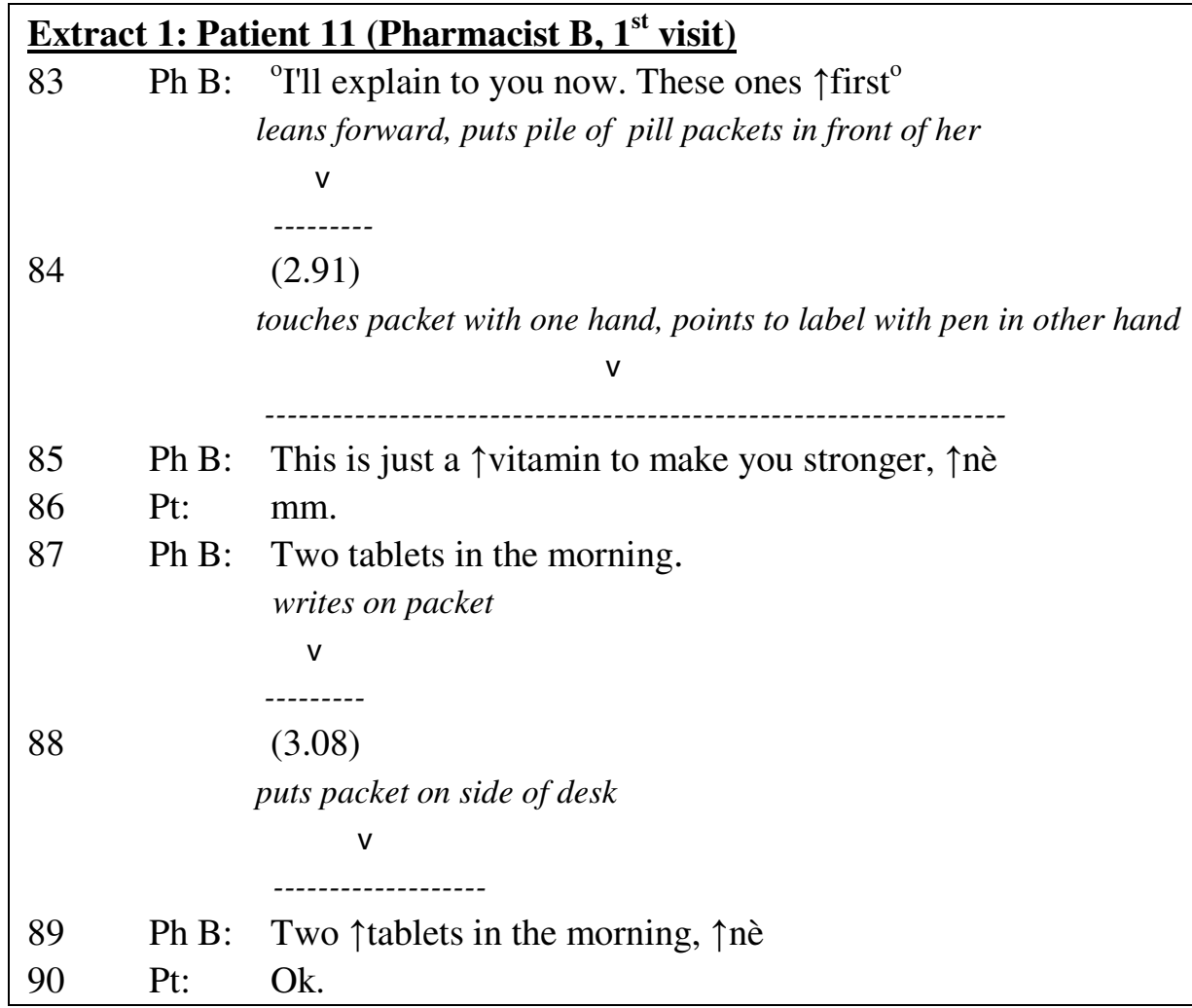




\subsection{Checking understanding}

The pharmacists' verification of patients' understanding of instructions formed an important part of each interaction and various strategies were used by pharmacists to attempt to ensure that patients understood dosage instructions (discussed in more detail in Watermeyer and Penn 2009b).

One strategy used continually in the interactions was the elicitation of a demonstration of understanding from patients (by using an open-ended request for the patient to "tell" or "show" the pharmacist how they will take or are taking their ARV medications), rather than relying on a mere assertion of understanding. As Pilnick (1999) points out, an actual demonstration of understanding is preferable to an assertion of understanding. By issuing such a request, patients were required to demonstrate their understanding and the pharmacists were able to ascertain directly whether the patients had understood the dosage instructions for each drug.

For example, in Extract 2, after the first request for a demonstration of understanding (line 142) the pharmacist is able to ascertain that the patient understands some of the information but that she does not understand that only Nevirapine must be taken in the morning only for the first fifteen days and not the other two ARVs (line 143). The pharmacist quickly identifies this misunderstanding and immediately initiates clarification of the instructions (line 144). Later in the interaction, a second request for a demonstration of understanding (lines 189, 191) reveals that the patient now understands and further clarification or repetition of instructions is not necessary.

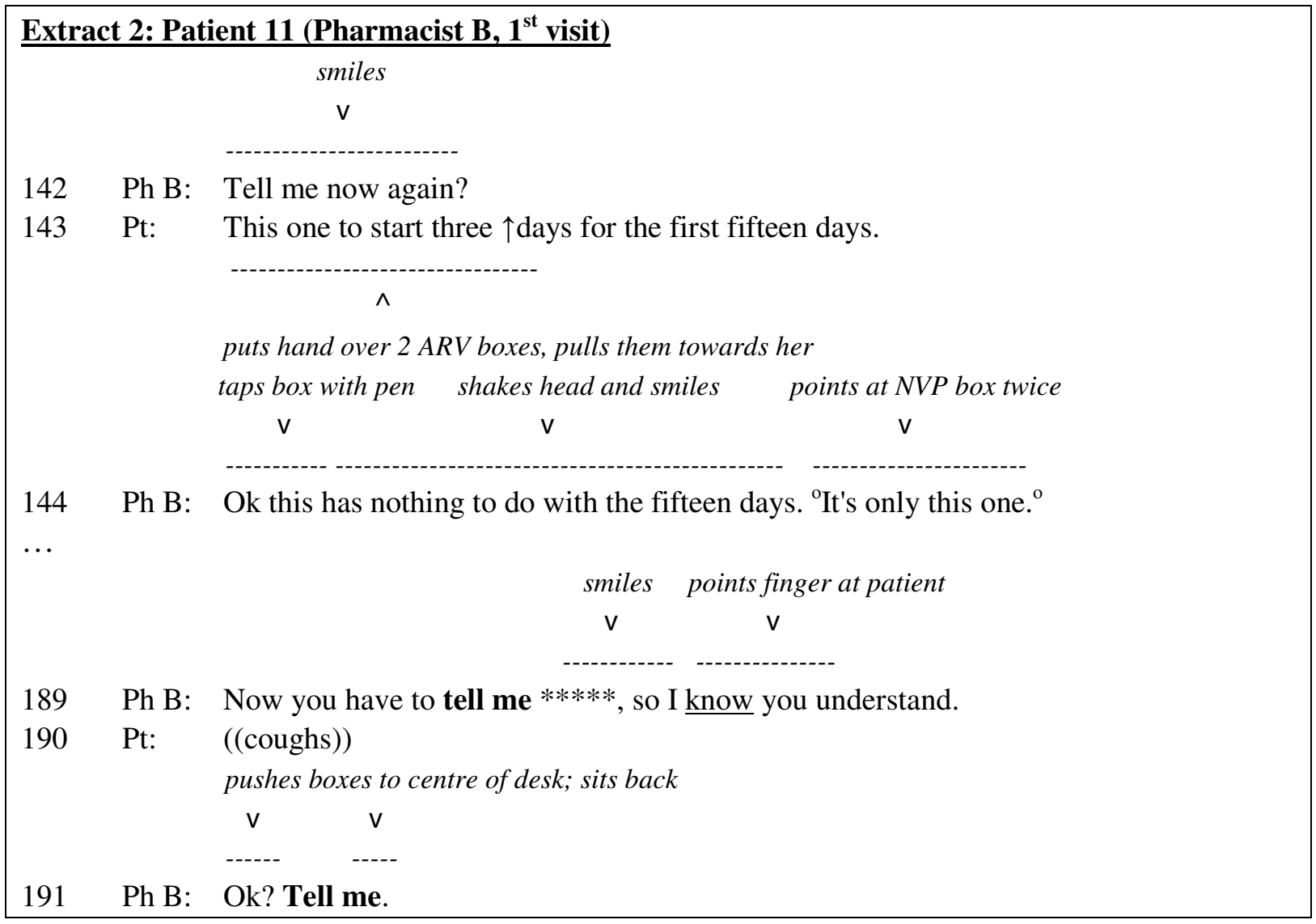


Pilnick's (1999) research revealed reluctance on the part of pharmacists to request such a demonstration, for fear of insulting or attacking patients' competence (Pilnick 2003). Pharmacists in her data appeared to rely on an assumption of patients' implicit understanding, on indirect demonstrations of understanding by patients, or on assertions of understanding. In this study, however, the pressure felt by the pharmacists to ensure understanding of the ARV dosage instructions despite linguistic barriers appeared to be a driving factor which superseded any possible insult that patients may have felt.

In addition to requesting a demonstration of understanding, pharmacists employed certain indirect techniques to verify patient understanding, including the frequent use of questions which required a simple yes/no assertion from the patients. These questions were usually a variation of do you understand? (e.g. Extract 3, lines 168 and 170). However, this method obtained only an assertion of understanding and did not elicit a demonstration of comprehension.

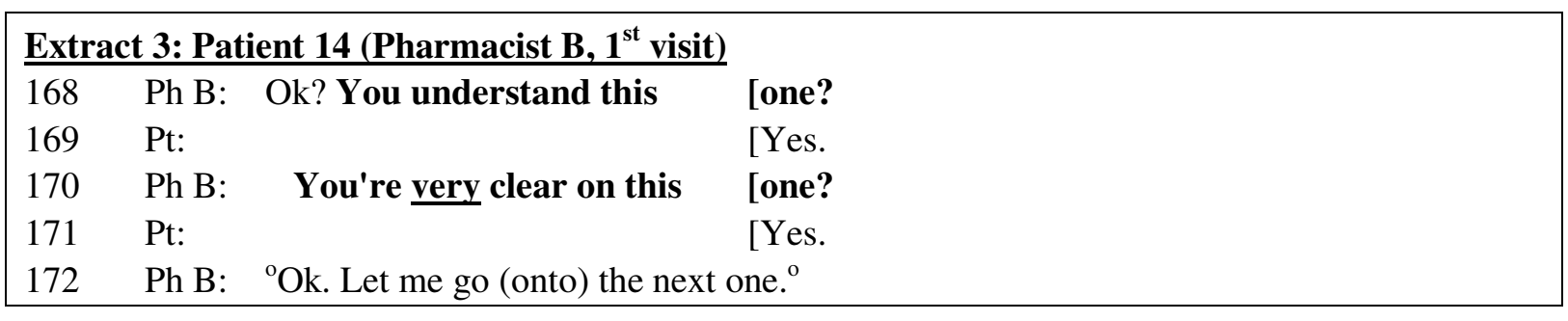

In cross-cultural interactions and institutional interactions in general, patients may respond positively to such questions out of politeness or deference to the authority of the health professional, making this an unreliable verification technique (Cass et al. 2002; Meeuwesen et al. 2007). Therefore, this strategy may not provide pharmacists with a true reflection of patients' understanding of instructions.

Another common technique involved the use of so-called "response solicitations" in a tag question position, such as nè?, ok?, and isn't it? Generally, tag questions are used pragmatically to express a speaker's uncertainty or they may serve a general politeness function by inviting the listener to join the discourse (Brown and Levinson 1987). However, in these interactions they appeared to serve as response solicitations (Jefferson 1981) - i.e. they requested a response from patients to information or instructions presented - and they also served to check whether patients had understood information and were following the discussion and to invite patients to request clarification of information. For example, in Extract 1, the pharmacist uses a response solicitation (line 89) to obtain a response from the patient (line 90) which indicates that she is following the pharmacist's explanation and that she does not require clarification of information.

When asked what they understood about the ARVs and the dosage instructions, most patients were able to relate basic instructions such as that the pills must be taken twice a day at the same time, the pills should not be taken with alcohol or fatty foods, as well as the importance of adherence. A few patients mentioned that ARVs do not cure HIV/Aids but that they boost the immune system and decrease the viral load. Interestingly, even those patients who 
indicated that they struggled to understand English or Afrikaans appeared to understand basic information about how to take the ARVs.

\subsection{Non-verbal behaviours}

During transcription of the data, it was obvious to the researchers that non-verbal behaviours such as eye gaze, body language and posture, facial expression, gesture, prosody and specifically the use of props constitute vitally important components of each interaction. Nonverbal behaviours are integral to the meaning of communication and cannot be separated from the content of the interactions (Goldin-Meadow and Wagner 2005). Non-verbal behaviours are also of particular importance in cross-cultural interactions and often constitute politeness behaviours (Kasanga and Lwanga-Lumu 2007).

Non-verbal strategies included the following (described in more detail in Watermeyer and Penn, 2009a):

- Visual demonstration and the use of props (pill boxes and containers, labels, brown paper bags, diary cards). The use of these strategies served to reinforce explanations and provided communicative realism. Patients were able to use props to demonstrate understanding of information across language barriers.

- Verbal and non-verbal demarcation of drugs, especially the separation of ARVs from other drugs (as in Extract 1). As the pharmacists talked about a specific drug, they moved the relevant boxes and containers to the centre of the desk or towards the patient and the other boxes were moved to the side. This strategy ensured that patients knew which drug was being discussed and this helped to eliminate any potential confusion while pharmacists provided dosage instructions.

- Supplementation of verbal instructions by pointing to each medication container while giving an instruction. For instance, while giving ARV dosage instructions, the pharmacists pointed to each box and then gave an instruction. By doing this, the pharmacists provided a visual reinforcement of their verbal instructions.

- Opening boxes or containers to show pills to patients. On several occasions, pharmacists actually cut the pill sheets into single pills in order to demonstrate dosage instructions. When this strategy was used, pharmacists were careful to ensure that they placed each pill on top of the relevant container so that patients could associate the particular pill with its corresponding container.

- Various types of gestures were used by pharmacists to supplement instructions and by patients to demonstrate their understanding. These included symbolic (e.g. giving a "thumbs up" sign), deictic (pointing to pill pots), iconic (e.g. pointing to the chest), pantomimic (e.g. to demonstrate how to use a syringe) and interactive gestures (various non-specific hand shapes used throughout conversations, usually for emphasis).

- Body posture was used to signal turn taking shifts or the end of turns.

- Emphasis of information and instructions using combinations of varied intonation, pauses, slower speech rate, softer speech, code switching, gesture and posture.

- Eye gaze which established and maintained joint attention.

- Careful monitoring of patients' non-verbal responses which provided clues that patients had not understood explanations or were not following explanations.

Interestingly, patients often used the pill boxes and containers as props, usually when demonstrating their comprehension of dosage instructions or when telling the pharmacist how 
they took their ARVs. Patients appeared to find it easier to explain by pointing to the relevant container, as they did not always know the names of the drugs but rather relied on what the container or the actual pill looked like. Even those patients who were not fluent English speakers were able to demonstrate their understanding simply by pointing to the containers and using a few English words mixed with Setswana phrases to explain when they took the pills.

Extract 4 illustrates how a patient is able to use the props to demonstrate his understanding of the dosage instructions. Although this patient reported that he is proficient in English, he uses minimal language when explaining and instead relies on the props. It is easier for him simply to point to the relevant boxes (e.g. line 25) than to use the rather lengthy and complex drug names. The pharmacist knows exactly what the patient is trying to say (line 28) and he is able to demonstrate his understanding of the dosage instructions clearly.

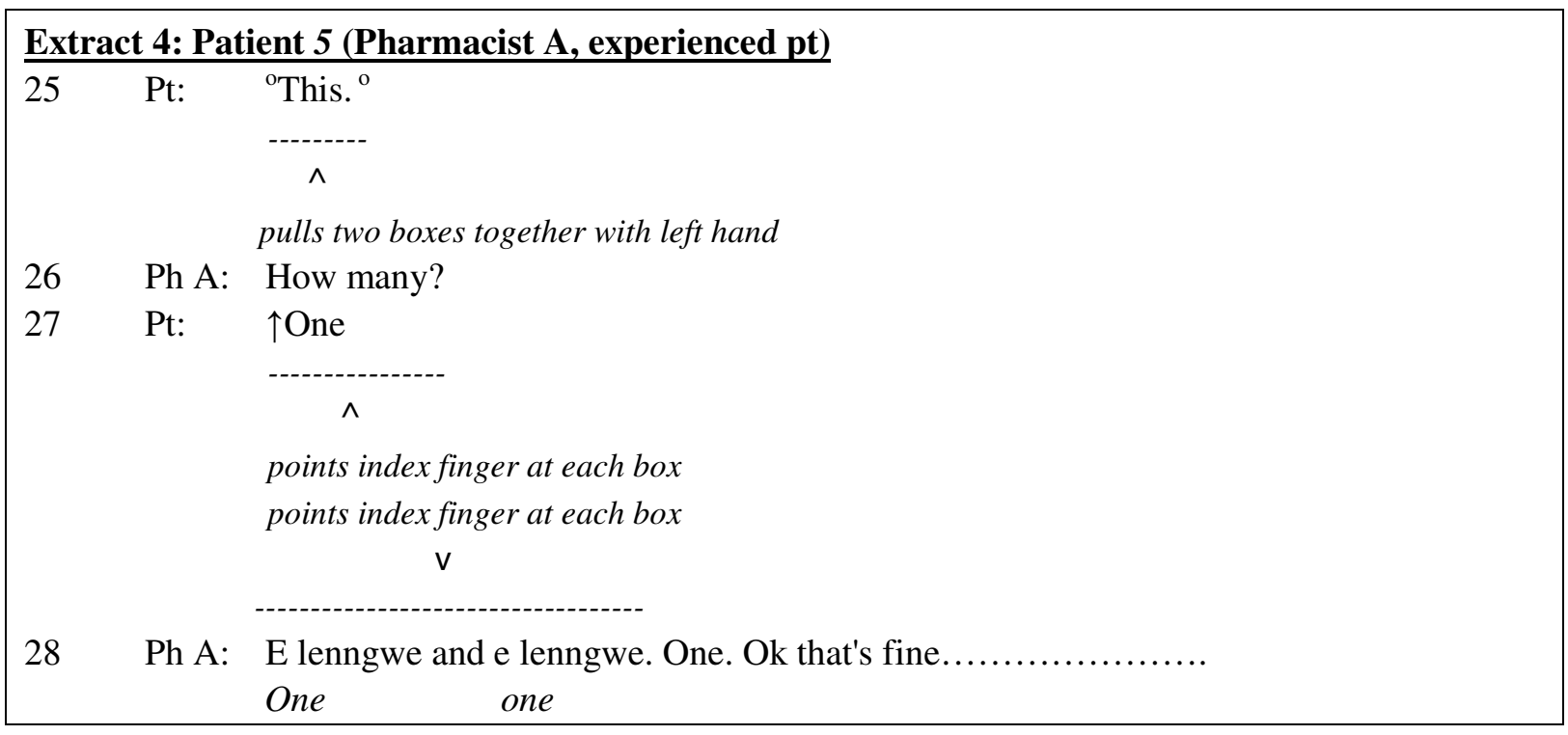

On many occasions across the data corpus, pharmacists employed a number of prosodic and non-verbal techniques to emphasise certain instructions or to get patients' full attention prior to delivering information. These techniques included the use of stress, the patient's name, pauses, slowed speech rate, softer speech, code switching, rising intonation, gesture and a "conspiratory" posture (i.e. the pharmacist leant forward towards the patient).

In Extract 5, the pharmacist picks up a pill pot and holds it up for the patient to see. Just before she begins to give the instruction, she leans forward in a "conspiratory" posture and her facial expression becomes rather serious, as if to prepare the patient for the important information that will follow (line 233). She lowers the pot and clearly circles the pot label to show the patient that the dosage instruction is written on the pot. She gives the instruction in Setswana and repeats it in English, stressing the word only, using gesture and a serious facial expression to supplement this important verbal instruction (line 234). 


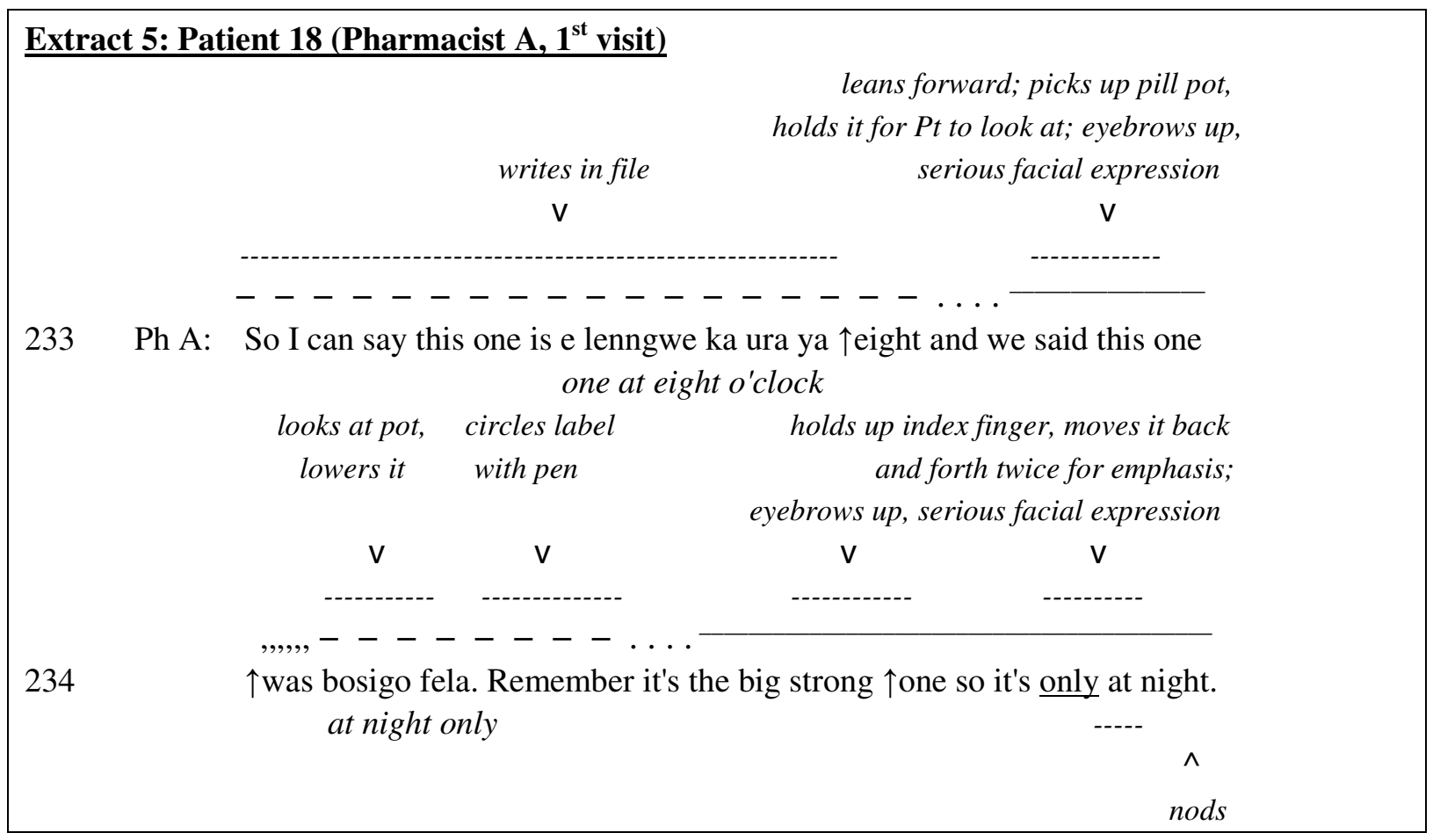

\subsection{Rapport}

A strong feature of the interactions was the pharmacists' use of opportunities to create rapport with patients and various strategies were used by pharmacists to achieve this. The establishment and maintenance of rapport is considered an important objective of any patienthealth professional relationship, because it encourages the development of trust within this relationship. Rapport may improve patient satisfaction with health care services and achievement of treatment outcomes and it may well increase treatment adherence (Fiksdal 1988).

Several pharmacist behaviours which appeared to contribute towards encouraging rapport included the following:

- Encouragement and reassurance, often in response to patients' anxiety and apprehension about the ARVs or uncertainty about understanding of dosage instructions.

- Actively listening to patients.

- Showing a personal interest in patients' lives, e.g. asking about their birthday or their grandchildren.

- Exploring patients' health and emotional concerns.

- Responding to patients' fears, feelings and concerns.

- Showing empathy towards patients and their situations.

- Using non-verbal strategies such as smiling, leaning forward, head nodding, increased gaze behaviours, or physical touch.

Extract 6 illustrates an empathetic response by a pharmacist to a patient's expression of emotion. The pharmacist has finished explaining dosage instructions to the patient. The patient then makes a comment (line 142) which is emotionally laden and conveys much about how she 
is feeling. The patient is clearly concerned about her ability to adhere to the regimen and understand the instructions correctly and she appears to doubt her own ability to do this without assistance. She also indirectly expresses how difficult this process is for her and how she needs support - perhaps both emotionally and physically - while taking the ARVs. The pharmacist senses the patient's anxiety and she responds initially with a practical solution i.e. that the patient can turn to her grandchild for support (line 143). The pharmacist then initiates an empathetic, kind response to the patient's expression of concern by reassuring her both verbally and non-verbally (line 144).

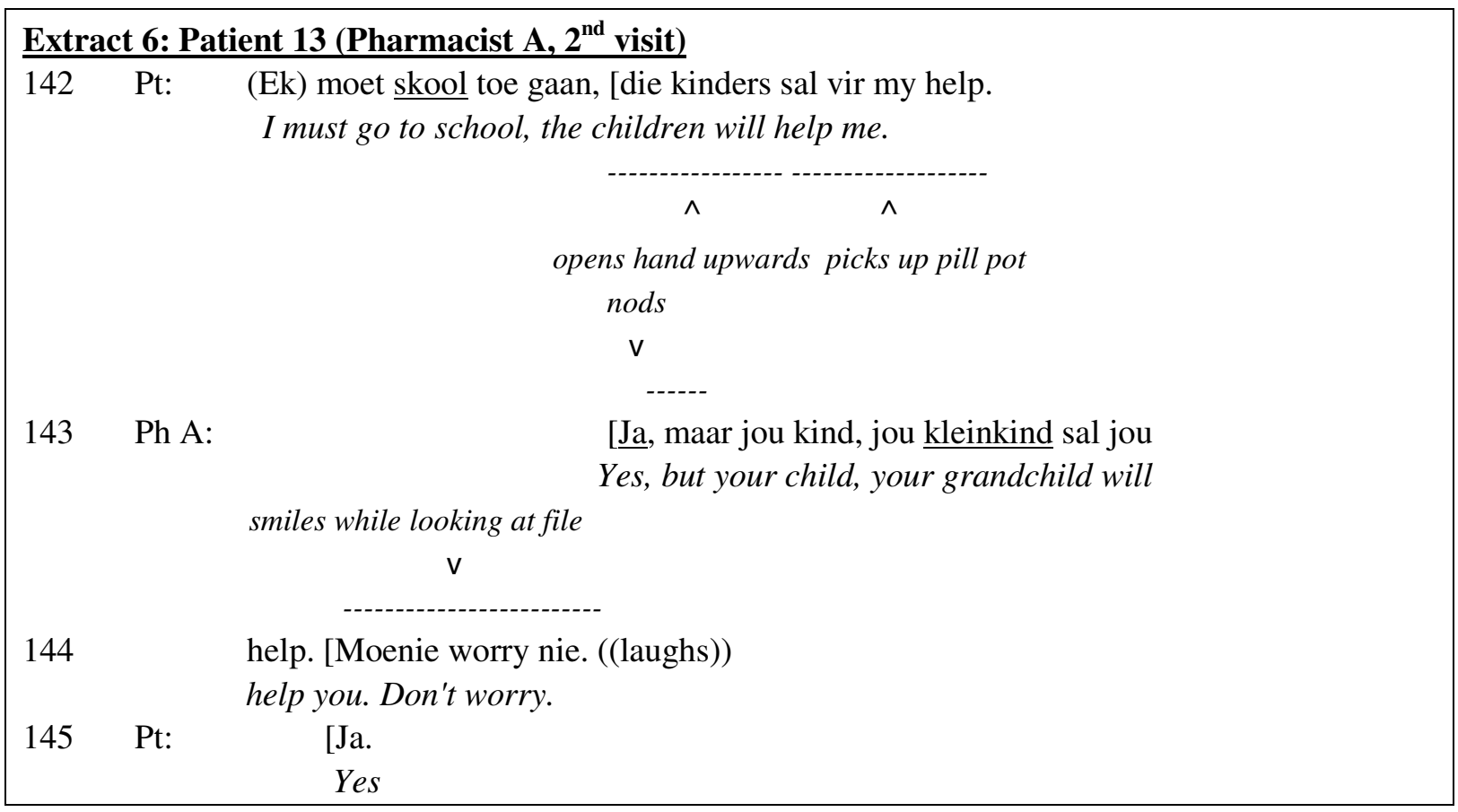

\subsection{Collaboration}

Many of the strategies employed by the pharmacists appeared to foster collaboration between themselves and patients. According to Leach (2005:264), "a collaborative consultation style ... is essential to building a therapeutic relationship". Through encouraging collaboration with a health professional, patients may be empowered to participate in their own care. In order to promote collaboration, health professionals need to adopt a patient-centred approach to interactions and focus on developing mutually agreed goals together with the patient - in the pharmacy context, that goal is principally to promote understanding of dosage instructions (which may directly impact upon adherence behaviours). A collaborative consultation style also involves effective communication skills such as using open-ended questions, reflecting on what the patient says, and paraphrasing and summarising information.

Collaborative strategies and processes identified in the data corpus included the following:

- Pharmacists and patients engaged in successful repair sequences, especially when patients initiated clarification requests.

- Patients volunteered information about themselves or voiced concerns regarding their health.

- Patients asked questions about the medicines. 
- Patients initiated or volunteered a demonstration of their understanding of the dosage instructions.

- Patients contributed comments during the interactions.

- Patients alerted pharmacists to errors in prescription or dispensing or to confusion over generic medicines.

- Patients initiated personal moments (e.g. I'm tired).

Extract 7 provides an illustration of collaboration between pharmacist and patient as they initiate a repair sequence in an effort to understand the dosage instructions. Prior to the start of this extract, the pharmacist has asked the patient to describe how he is taking his tablets. He begins (lines 31,33), but the pharmacist soon notices that there has been a misunderstanding and she requests clarification from the patient (line 34). The patient then realises that he does not recognise one of the drugs. He reaches out to pick up the pill pot and simultaneously asks the pharmacist for the name of the drug (line 35). The pharmacist consults the patient's file to determine the cause of the misunderstanding (line 36). The patient then volunteers information about his regimen: he takes vitamins, not this "unknown" drug (line 37). The pharmacist corrects him and explains that this is the prescribed drug (line 38) and he hands across the pill pot for the pharmacist to scrutinise (line 39).

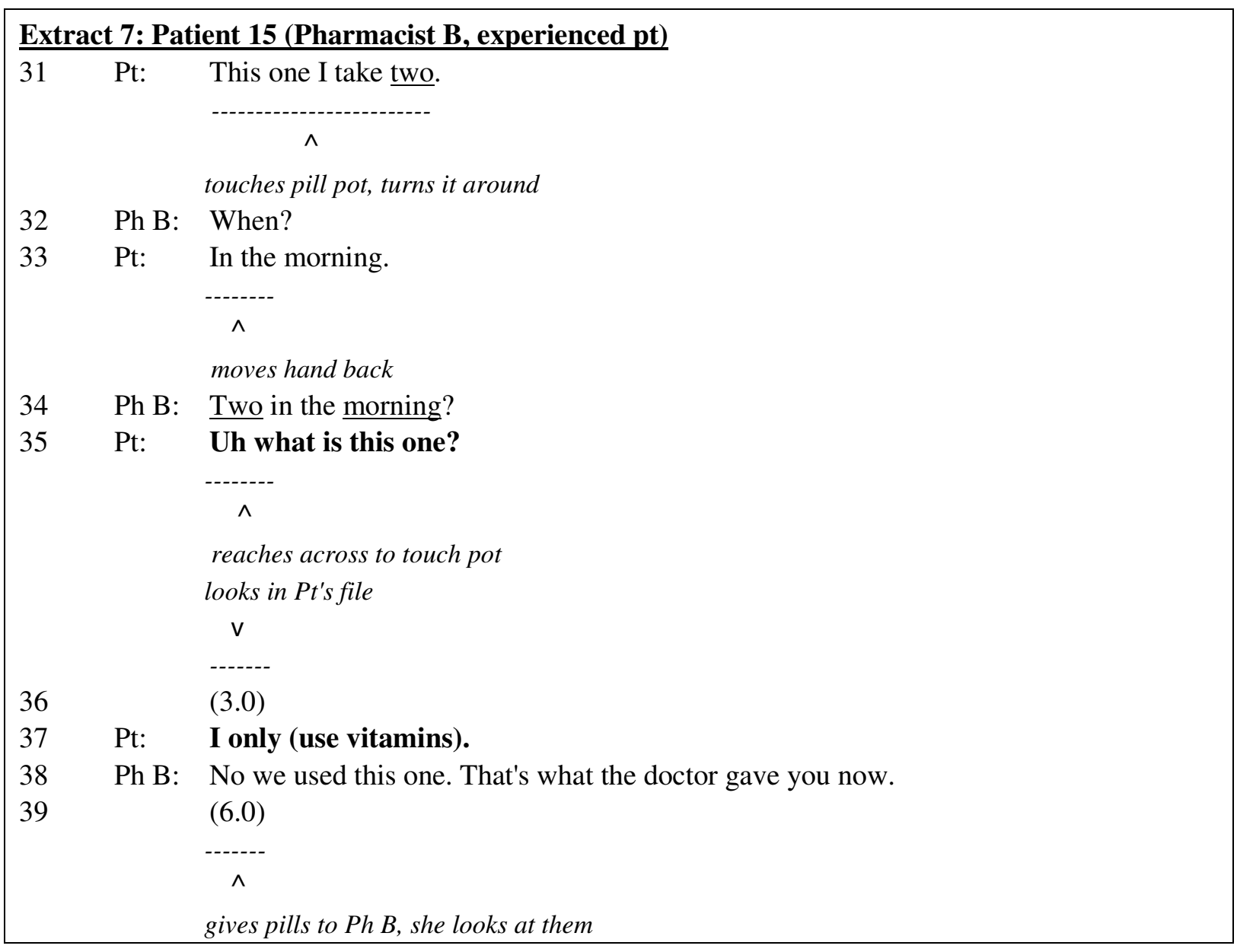

There is a definite sense of collaboration between the two parties as they work towards solving the problem. Interestingly, at times during this interaction, this patient is not 
particularly responsive to the pharmacist and a sense of rapport is not always present. Despite this, the patient appears to feel comfortable enough in the situation to initiate requests for clarification and other information.

\section{Discussion and conclusions}

The interactions described in this paper have a clearly demarcated structure within which there are many interactive verbal and non-verbal strategies which are utilised by both pharmacists and patients in order to achieve the collaborative goals of understanding of the ARV dosage instructions. What is apparent is the sensitivity of pharmacists towards each patient's communicative needs and their ability to adapt their communication style accordingly. It is in the minute details of communication that we are shown how, despite barriers of context, culture and language, patients and pharmacists appear able to achieve positive, successful, collaborative interactions. This study suggests that one cannot assume that a profoundly difficult context will necessarily hamper communication between health professionals and patients.

It is obvious that there are certain differences between the pharmacist-patient interactions in this context and elsewhere. Although only a small number of published studies exist, these indicate that pharmacist-patient interactions are not typically collaborative and pharmacists do not encourage patient participation, even when there are no language and cultural barriers. However, the data presented in this paper suggests that pharmacy interactions can be efficient and collaborative, patients can be encouraged to participate actively in interactions and communication can be successful even across cultural and language barriers. The fact that patients initiated clarification requests and participated actively in consultations with the pharmacists is particularly encouraging in light of previous studies that indicate that this does not typically occur in pharmacy interactions. It would appear that the global move towards consumerism in health care, as well as the urgency presented by HIV/Aids, are promoting a change in these past influences of patient behaviours and patients are now encouraged to be active agents in their own health situations.

The daily interface of different cultures and languages within the South African environment and the normality of communicating across barriers are possible reasons for the differences between this study and other research. When two cultures meet, a natural process of crosscultural adaptation inevitably occurs (Kim 2001). In order to achieve effective communication across cultural barriers and meet role requirements in specific situations, people adapt their interactive styles and may reciprocate the style of the conversational partner (Burgoon, Stern and Dillman 1995). The evidence in this study suggests that through a process of adaptation, pharmacists and patients may have been able to transcend some of the barriers to communication and collaboration that have been identified in other studies.

This study also suggests that the influence of the historical context of segregation has heightened awareness of differences between cultures. Recent moves towards reducing inequalities and racial boundaries in South Africa may have encouraged participants to incorporate the norms of other cultures into their communicative style - for instance, pharmacists have adopted Tswana forms of address and patients initiate clarification requests which appear to transgress cultural norms of not questioning authority. The results are 
therefore particularly interesting and imply that the diversity of South Africa provides a resource that can inform training, policy and future practice both locally and worldwide.

The profound urgency of the disease, the intense pressures and anxieties associated with the need to adhere strictly to a life-prolonging treatment to ensure success and the resultant pressure felt by pharmacists to promote patient understanding also appear to act as driving factors within the interactions. Stein, Lewin and Fairall (2007:961) note that "the long wait for ART [in South Africa] appears to have heightened the sense of urgency and enthusiasm for implementation". Several strategies, for example checking understanding and the use of response solicitations, appear to have evolved out of this urgency and the need to ensure that patients understand information and dosage instructions related to ARVs and HIV/Aids. Patients have also adopted communication behaviours such as repair strategies, perhaps in recognition of the fact that they are responsible for ensuring understanding so that they are able to adhere to ARV treatment.

The results of this study can be used to inform training, policy and future pharmacy practice in the context of HIV/Aids, both locally and worldwide. The process of globalisation means that pharmacists (and other health professionals) are increasingly faced with cultural and linguistic barriers and interpreters are not always available to assist with overcoming these barriers (Flores 2006). Therefore, there is a greater need for the inclusion of communication skills training as a component of pharmacist training to enable pharmacists to improve service provision across cultural and linguistic barriers and modify their communication or implement a few interactive strategies in order to promote improved understanding across language barriers. By becoming aware of potential facilitators and inhibitors to communication, as well as the range of contextual and process variables that may affect patients' comprehension of dosage instructions, interactions between pharmacists and patients have the potential to become more efficient.

While this is a South African study conducted within a specific context, it informs other global and multicultural issues in the pharmacy profession. In response to the call for the provision of communication and counselling skills training for pharmacists and the need for pharmacists to acquire such skills (Salter et al. 2007), training programmes need to be developed, evaluated and implemented. It is the researchers' belief that given the results of this study, such programmes should take cognisance of site-, context- and disease-specific needs as well as multicultural and multilingual issues. In particular, this study demonstrates the value of this method of detailed interactional analysis in identifying how instructions are given to patients, how understanding is verified, as well as potential facilitators and barriers to communication and the communication competencies required by pharmacists.

While an opportunity to track the patients included in this study across multiple visits would have been advantageous in order to ascertain whether correct understanding of the dosage instructions and positive adherence behaviours were maintained over time, this was not possible for this study and is seen as a limitation which could be addressed in future studies. Nonetheless, the description of cross-cultural pharmacy interactions which is presented in this paper addresses a gap in the literature and provides a valuable resource which can be used to improve communication in pharmacy practice, both locally and worldwide. 


\section{Acknowledgements}

The authors would like to thank the patients and pharmacists who participated in this research.

This study was supported by a grant from the National Research Foundation (NRF).

\section{Note}

1. Nè is an Afrikaans word. It means "isn't it?", "not so?" or "right?".

\section{References}

Aronson, J. 1994. A pragmatic view of thematic analysis. The Qualitative Report 2: 1-4.

Brown, P. and S. Levinson. 1987. Politeness: Some universals in language usage. Cambridge: Cambridge University Press.

Burgoon, J., L. Stern and L. Dillman. 1995. Interpersonal adaptation: Dyadic interaction patterns. Cambridge: Cambridge University Press.

Cass, A., A. Lowell, M. Christie, P. Snelling, M. Flack, B. Marrnganyin and I. Brown. 2002. Sharing the true stories: improving communication between Aboriginal patients and healthcare workers. Medical Journal of Australia 176(10): 466-470.

De Young, M. 1996. A review of the research on pharmacists' patient-communication views and practices. American Journal of Pharmaceutical Education 60: 60-77.

Dyck, A., M. Deschamps and J. Taylor. 2005. Pharmacists' discussions of medication side effects: a descriptive study. Patient Education and Counseling 56(1): 21-27.

Fiksdal, S. 1988. Verbal and nonverbal strategies of rapport in cross-cultural interviews. Linguistics and Education 1: 3-17.

Flores, G. 2006. Language barriers to health care in the United States. The New England Journal of Medicine 355(3): 229-231.

Goldin-Meadow, S. and S. Wagner. 2005. How our hands help us learn. Trends in Cognitive Sciences 9(5): 234-241.

Gross, R., W. Bilker, H. Friedman and B. Strom. 2001. Effect of adherence to newly initiated antiretroviral therapy on plasma viral load. AIDS 15(16): 2109-2117.

Hardy, H. 2005. Adherence to antiretroviral therapy: the emerging role of HIV pharmacotherapy specialists. Journal of Pharmacy Practice 18(4): 247.

Heath, C. 1986. Body movement and speech in medical interaction. Cambridge: Cambridge University Press.

Heritage, J. 1993. Conversation analysis and institutional talk: Analysing data. In D. Silverman (ed.) Qualitative Research: Theory, Method and Practice. London: Sage Publications. pp. 161-182.

Jefferson, G. 1981. The abominable ne?: An exploration of post-response pursuit of response. In P. Shroder (ed.) Sprache der Gegenwaart. Düsseldorf: Pedagogischer Verlag Schwann. pp. 53-88.

Jefferson, G. 2004. Glossary of transcript symbols with an introduction. In G. Lerner (ed.) Conversation Analysis: Studies from the first generation. Philadelphia: John Benjamins. pp. 13-23.

Kansanaho, H. 2006. Implementation of the principles of patient counselling into practice in Finnish community pharmacies. Ph.D. dissertation, University of Helsinki, Helsinki, Finland.

Kasanga, L. and J. Lwanga-Lumu. 2007. Cross-cultural linguistic realization of politeness: A study of apologies in English and Setswana. Journal of Politeness Research 3: 65-92.

Kim, Y. 2001. Becoming intercultural: an integrative theory of communication and cross- 
cultural adaptation. Second Edition. Thousand Oaks: Sage Publications.

Leach, M. 2005. Rapport: A key to treatment success. Complementary Therapies in Clinical Practice 11: 262-265.

Meeuwesen, L., F. Tromp, B. Schouten and J. Harmsen. 2007. Cultural differences in managing information during medical interaction: How does the physician get a clue? Patient Education and Counseling 67(2): 183-190.

Paterson, D., S. Swindells, J. Mohr, M. Brester, E. Vergis, C. Squier, M. Wagener and N. Singh. 2000. Adherence to protease inhibitor therapy and outcomes in patients with HIV infection. Annals of Internal Medicine 133: 21-30.

Patton, M. 1990. Qualitative Evaluation and Research Methods. Second Edition. London: Sage Publications.

Penn, C. 2007. Factors affecting the success of mediated medical interviews in South Africa. Current Allergy and Clinical Immunology 20(2): 66-72.

Pilnick, A. 1998. "Why didn't you say just that?" Dealing with issues of asymmetry, knowledge and competence in the pharmacist/client encounter. Sociology of Health and Illness 20(1): 29-51.

Pilnick, A. 1999. Patient counselling by pharmacists: Advice, Information or Instruction? The Sociological Quarterly 40(4): 613-622.

Pilnick, A. 2001. The interactional organization of pharmacist consultations in a hospital setting: A putative structure. Journal of Pragmatics 33(12): 1927-1945.

Pilnick, A. 2003. "Patient counselling" by pharmacists: four approaches to the delivery of counselling sequences and their interactional reception. Social Science and Medicine 56(4): 835-849.

Sacks, H. 1984. Notes on methodology. In J. Atkinson and J. Heritage (eds). Structures of social action: Studies in Conversation Analysis. Cambridge: Cambridge University Press. pp. 21-27.

Salter, C., R. Holland, I. Harvey and K. Henwood. 2007. "I haven't even phoned my doctor yet." The advice giving role of the pharmacist during consultations for medication review with patients aged 80 or more: qualitative discourse analysis. British Medical Journal 334: 1101-1107.

Shah, M., S. King and A. Patel. 2004. Intercultural disposition and communication competence of future pharmacists. American Journal of Pharmaceutical Education 68(5): 111.

Skoglund, P., D. Isacson and K. Kjellgren. 2003. Analgesic medication - communication at pharmacies. Patient Education and Counseling 51: 155-161.

Sleath, B., D. Roter, B. Chewning and B. Svarstad. 1999. Asking questions about medication: Analysis of physician-patient interactions and physician perceptions. Medical Care 37(11): 1169-1173.

Stein, J., S. Lewin and L. Fairall. 2007. Hope is the pillar of the universe: Health-care providers' experiences of delivering anti-retroviral therapy in primary health-care clinics in the Free State Province of South Africa. Social Science and Medicine 64: 954-964.

ten Have, P. 2000. Doing Conversation Analysis: A practical guide. London: Sage Publications.

Watermeyer, J. 2008. Pills of wisdom: An investigation of pharmacist-patient interactions in a South African antiretroviral clinic. Unpublished Ph.D. thesis, University of the Witwatersrand, Johannesburg, South Africa. 
Watermeyer, J. and C. Penn. 2009a. "Come, let me show you": The use of props to facilitate understanding of antiretroviral dosage instructions in multilingual pharmacy interactions. In L. Lagerwerf, H. Boer and H. Wasserman (eds). Health Communication in Southern Africa: Engaging with Social and Cultural Diversity. Amsterdam: Rozenberg Publishers. pp. 191-216.

Watermeyer, J. and C. Penn. 2009b. "Tell me so I know you understand": Pharmacists' verification of patients' comprehension of antiretroviral dosage instructions in a crosscultural context. Patient Education and Counseling 75: 205-213.

Watermeyer, J. and C. Penn. 2009c. The organization of pharmacist-patient interactions in an HIV/Aids clinic. Journal of Pragmatics 41: 2053-2071.

Wooffitt, R. 2005. Conversation Analysis and Discourse Analysis: A comparative and critical introduction. London: Thousand Oaks.

\section{Appendix}

$\mathrm{Ph}$ : Pharmacist (A or B)

\section{Key to Extracts}

Pt: Patient

$\underline{\text { word }}$

((laugh))

?

.

(.)

$(0.0)$

(word)

${ }^{\mathrm{o}}$ word ${ }^{\mathrm{O}}$

[ ]

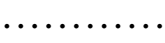

$=$

$-$

$\uparrow$

hhh

$* * *$

Conversation Analysis Transcription Conventions (Jefferson 2004)

some form of stress, via pitch or amplitude

transcriber's descriptions, e.g. laughter or head nod

rising intonation

falling or terminal intonation

continuing intonation

short pause

elapsed time in silence by tenth of seconds

especially dubious hearings or speaker identifications

softly spoken, quieter than the surrounding talk

onset and offset of overlapping talk

omitted text

latching (no gap between lines)

prolongation of the immediately prior sound

cut-off

shift into higher pitch in the utterance immediately following the arrow out breath or sigh

text omitted to protect participant's confidentiality

\section{Transcription system for vocal and visual elements (Heath 1986)}

Where there are two speakers, one must be placed above the other. In this case, the pharmacists' non-verbal behaviours are transcribed above their talk and the patients' below their talk.

A continuous line indicates that the participant is gazing at the face of the coparticipant. 
${ }_{-}-$A $_{\text {- }}$ A series of longer dashes indicates that the participant is looking at a specific object.

,,,,,,,$\quad$ A series of commas indicates that the participant is turning away from a coparticipant (or object).

$\ldots \ldots$ A series of dots indicates that the participant is turning towards a co-participant (or object).

A series of close dashes indicates movement (e.g. gesture, body posture, facial expression, head nodding), in relation to where the movement begins and ends in the talk. The dashes are accompanied by a description of the movement.

$\wedge$

movement

or

movement Indicates movement by pharmacist.

Indicates movement by patient.

$\mathrm{v}$ 